\title{
Static, Dynamic and Pogressive Collapse Analysis of Multi Storey (G+10) Residential Building by ETABS Software
}

\author{
PonnanaRamprasad, MadhusmitaMoharana, Ch. Chandra Mouli
}

\begin{abstract}
This project presents an attempt to do static, dynamic and progressive collapse analysis of multistory $(G+10)$ residential building by ETABS (Extended 3D Analysis of Building Systems). ETABS is software that helps to anatomization and design of low and high-rise buildings and frame structures. In this project G+10 RC frame building is analysis statically (linear method) and dynamically (Response Spectrum method) along with Progressive Collapse analysis. All the members of the project are analyzed as per Indian codes IS 456:2000, IS 800:2007, and IS 1893:2002 (part1) code using this software. Here the result for Story stiffness, Base shear, Story Shear, Overturning moments, Maximum displacement, and Story Drift is compared between static and dynamic results for Zone2-(case1), Zone3-(case2), Zone4-(case3), Zone5-(case4) with medium soil type and for Progressive Collapse analysis

GSA guidelines are followed.

As per GSA guidelines three column removal cases for each case1, case2, case3, and case4 individually studied, namely Corner column removal, Exterior column removal and interior column removal at ground floor. For all three cases linear analysis study has been undertaken and DCR ratios are evaluated. Member having DCR ratio greater than 2 will going to fail for corresponding column removal case.
\end{abstract}

Key Words: Static analysis, Dynamic analysis, Progressive collapse analysis, GSA Guidelines.

\section{INTRODUCTION}

For proper design of the building, the building should be check statically and dynamically under different load condition so; that the building is safe from different types of disaster which is likely to come after the construction of the building is over. Generally, we consider Self weight, dead loads, Live Loads, wind loads and Earthquake loads for Static and dynamic Analysis. However, after doing static and dynamic analysis also we not sure the structure is safe against local failure due to sudden loss of Column in the structure or abnormal loading like blast of cylinder or due to terrorist attack. So, we have move one step further and check our building against failure of Column at different location in ground floor as per General Service Administration (GSA) guidelines.

\subsection{GUIDELINES OF GSA}

\subsubsection{Facility security levels (FSL):}

Revised Manuscript Received on December 30, 2019.

PonnanaRamprasad, M.Tech(Ph.D), Department of Civil engineering, Assistant Professor, Aditya Institute of Technology \& Management, Tekkali, Amdhrapradesh.

MadhusmitaMoharana, M.Tech, Department of Civil engineering, Aditya Institute of Technology \& Management, K.Kotturu, India.

CH. Chandra Mouli, M.Tech(Ph.D), Department of Civil engineering, Snr. Assistant Professor, Aditya Institute of Technology \& Management, K.Kotturu, India.
The facility security level resolve to define the norms and process for determining the FSL of a federal facility, which categorizes facilities, depend on the analysis of several security-related facility factors, including its target attractiveness, as well as its value or criticality.

\subsubsection{FSL I \& II:}

Specified the low occupancy and risk level correlated with these types of facilities, progressive collapse design is not preferred for FSL I and II, irrespective of the number of floors.

\subsubsection{FSL III \&IV:}

These Guidelines are relevant to FSL III and IV buildings with four stories or more sustained from the lowest point of exterior grade to the highest point of elevation. Uninhabited floors such as mechanical penthouses or parking shall not be considered a story. It shall implement both the Alternate Path and Redundancy design procedures.

\subsubsection{FSL V:}

These Guidelines are used for all FSL V buildings regardless of number of floors. FSL V facilities shall implement the Alternate Path method for identification of vertical load resisting element removal area. Redundancy design procedures not required for FSL V facilities.

\section{LITRATURE REVIEW}

- Sana Fatema et.al (2016) ${ }^{12}$ have publish a journal on "Progressive Collapse of Reinforced Concrete" as per GSA guidelines using ETABS software in International Journal of Emerging Trends in Science and Technology for evaluation of Progressive collapse linear static method and nonlinear static method of analysis has been used and they have concluded that shear in beam is not critical in any case, Columns are also not critical in Progressive collapse. But by Linear static analysis and nonlinear static it was observed that beams are going to fail in flexure.

- GirumMindaye et.al $(2016)^{9}$ have publish a journal on "Seismic Analysis of a Multistory RC Frame Building in Different Seismic Zones" in International Journal of Innovative Research in Science, Engineering and Technology (IJIRSET)which compares the story Stiffness, Base shear, Lateral force, Story Shear, Story displacement, Overturning moments and story drift statically and dynamically for different Zones cases.

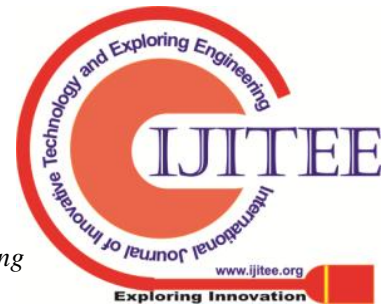




\section{Static, Dynamic and Pogressive Collapse Analysis of Multi Storey (G+10) Residential Building by ETABS \\ Software}

- B. Srikanth et al (2013) $)^{5}$ has published a journal on "Comparative Study of Seismic Response for Seismic Coefficient and Response Spectrum Methods" to analyze 20 storied building by both methods. In Response Spectrum Method, the time periods, natural frequencies and mode shape coefficients were calculated by MATLAB program then remaining process was done by manually. The modal combination rule for Response Spectrum Analysis was SRSS. The main parameters considered in that study was to compare the seismic performance of different Zones i.e. II and V were Base Shear, Story Moment and lateral forces.

\section{METHODOLOGY}

To understand the basic requirements such as safety, durability, economy, aesthetic appearance, feasibility, practicability and acceptability following methodology is followed.

\subsection{Study of different Zones and Soil data collection:}

\begin{tabular}{|c|c|}
\hline Zone & Intensity \\
\hline Zone V & $\begin{array}{l}\text { Very High Risk Zone Area } \\
\text { liable to shaking intensity } \\
\text { IX (and above) }\end{array}$ \\
\hline Zone IV & $\begin{array}{l}\text { High Risk Zone } \\
\text { Intensity VIII }\end{array}$ \\
\hline Zone III & $\begin{array}{l}\text { Moderate Risk Zone } \\
\text { intensity VII }\end{array}$ \\
\hline Zone II & $\begin{array}{l}\text { Low Risk Zone } \\
\text { VI (and lower) }\end{array}$ \\
\hline
\end{tabular}

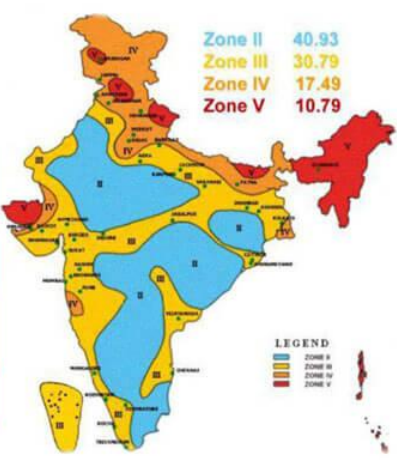

Fig 1.1 Seismic Zones of India

- $\quad$ For the analysis of the building, we have taken 4 cases in each Zone. The Soil bearing Capacity is assumed to be $200 \mathrm{KN} / \mathrm{m}^{2}$ with wind Speed of $50 \mathrm{~m} / \mathrm{s}$ along $\mathrm{X}$ direction $\left(0^{\circ}\right.$ and $\left.180^{\circ}\right)$ and along $\mathrm{Y}$ direction $\left(90^{\circ}\right.$ and $\left.270^{0}\right)$.

\subsection{Modeling and Loading:}

In ETABS first Modeling of multistory building which is to be analysis is done. In modeling material to be used to build the structure is defined. The input dates used for modeling are described below:

- Building type: G+10 Residential Building

- Plan area: $20(\mathrm{~m}) * 20(\mathrm{~m})$

- Beam size: $300(\mathrm{~mm}) * 300(\mathrm{~mm})$

- Column size: $350(\mathrm{~mm}) * 350(\mathrm{~mm})$

- Beam clear cover to Longitudinal Rebar Group centroid: $35 \mathrm{~mm}$

- Column clear cover to confinement Bars: $40 \mathrm{~mm}$

- Slab thickness: $150 \mathrm{~mm}$

- Typical story height: $3 \mathrm{~m}$

- Bottom story height: $3 \mathrm{~m}$

- Live load, LL: $3 \mathrm{kN} / \mathrm{m}^{2}$ External Wall load: $10.4 \mathrm{KN} / \mathrm{m}$

- Partition and floor finishing load, FL: $1.5 \mathrm{kN} / \mathrm{m}^{2}$

- Soil type: Type II (Medium Soil)

- Materials: M30 and Fe415 Grade

- Soil Bearing Capacity $200 \mathrm{KN} / \mathrm{m}^{2}$
- For Static and dynamic Analysis: Earthquake Direction: $\mathrm{X}$ and $\mathrm{Y}$ Seismic is defined

- Zone: II (Case 1) -Zone factor 0.10,

- Zone: III (Case 2)-Zone factor 0.16,

- Zone: IV (Case 3) -Zone factor 0.24 \&

- Zone: V (Case 4) -Zone factor 0.36

- For Progressive Collapse Analysis: In Each Cases further there are 3 Cases they are:

i) Corner Column Removal.

ii) Exterior Column Removal.

iii) Interior Column Removal.

- $\quad$ First a Response Spectrum function is defined for above 4 different Zones with Function Damping Ratio as 0.05 .

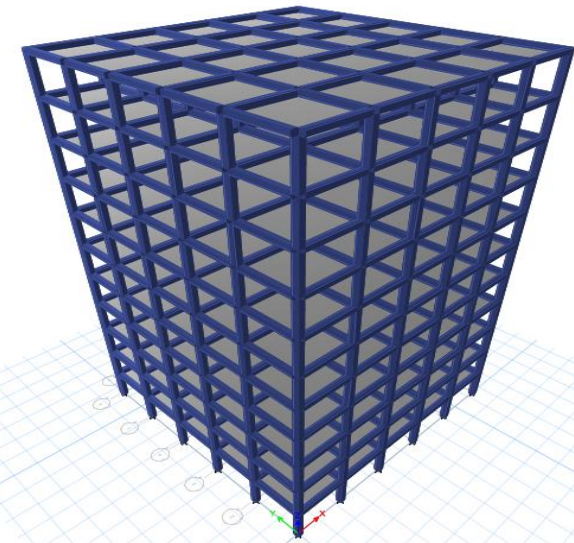

Fig 1.2 G+10 modeling using ETABS

\subsection{Comparison of Static and Dynamic output:}

After modeling and loading is done, the model is checked and design is done. The result for the for Story stiffness, Base shear, Story Shear, Overturning moments, Maximum displacement, and Story Drift is compared between static and dynamic results for Zone 2(case1), 3(case2),4(case3), 5(case4).

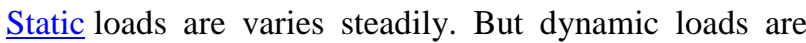
changes with time quickly in comparison to the structure's natural frequency. Due to change of loading steadily or quickly, the response of the structures varies static or dynamic analysis.

\subsection{For Linear Static PC Analysis:}

PCA by Linear Static is accomplishing as per General Service Administration (GSA) guidelines. Columns are removed methodically.. Here 3 case of column removal (Corner column, Exterior column and Interior column) are studied. Due to column removal cases identification of critical sections is done then DCR values are estimated. Because of high shear capacity DCR ratio is not evaluation for Shear of beam and in no case DCR of shear of beam will exceed more than one. Load combination as per GSA GLD $=2$ (1.2Dead Load + 0.5Live Load) for column removal region Combination and $\mathrm{G}=(1.2 \mathrm{DL}+0.5 \mathrm{LL})$ for other. 


\section{RESULT AND DISCUSSIONS}

The result evaluated for the Story Stiffness, Base Shear, Story Shear, Overturning Moment, Maximum Story displacement and Story Drift for Case1, Case2, Case3, Case 4 are shown in respective Table and Graph and with discussions.

\section{STORY STIFFNESS}

Table: 1 Story Stiffness in $\mathrm{X}$ and $\mathrm{Y}$ direction

\begin{tabular}{|c|c|c|c|c|}
\hline Story & $\begin{array}{c}\text { Static } \\
\mathbf{X}(\mathrm{K} / \mathrm{N} / \mathrm{m})\end{array}$ & $\begin{array}{c}\text { Dynamic } \\
\mathbf{X}(\mathrm{KN} / \mathrm{m})\end{array}$ & $\begin{array}{c}\text { Static } \\
\mathbf{Y}(\mathrm{K} / \mathrm{N} / \mathrm{m})\end{array}$ & $\begin{array}{c}\text { Dynamic } \\
\mathbf{Y}(\mathrm{K} / \mathrm{N} / \mathrm{m})\end{array}$ \\
\hline 1 & 294569.764 & 297471.346 & 295296.4 & $\mathbf{2 9 8 2 3 4 . 9 1}$ \\
\hline 2 & 196129.332 & 198186.872 & 196701.6 & 198757.71 \\
\hline 3 & 187733.35 & 188977.494 & 188193.4 & 189520.81 \\
\hline 4 & 185686.177 & 186720.833 & 186039.1 & 187145.82 \\
\hline 5 & 184483.879 & 185330.067 & 184734.5 & 185635.35 \\
\hline 6 & 183386.199 & 184234.671 & 183537.2 & 184431.39 \\
\hline 7 & 182179.331 & 183589.656 & 182220.1 & 183687.55 \\
\hline 8 & 180589.478 & 182872.451 & 180486.5 & 182863.11 \\
\hline 9 & 177905.304 & 182294.676 & 177576.1 & 182129.65 \\
\hline 10 & 170892.282 & 179021.728 & 170151.1 & 178553.51 \\
\hline 11 & 130156.086 & 139853.902 & 128911.5 & 138914.3 \\
\hline
\end{tabular}

Figure: 2.1Story Stiffness in $\mathrm{X}$ direction

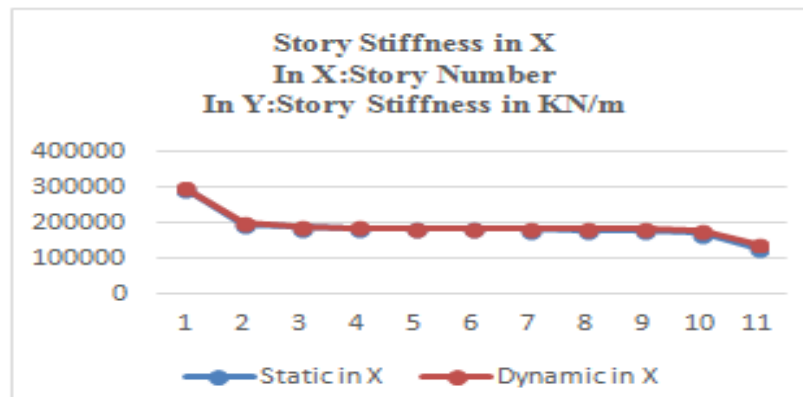

Figure: 2.2 Story Stiffness in Y direction

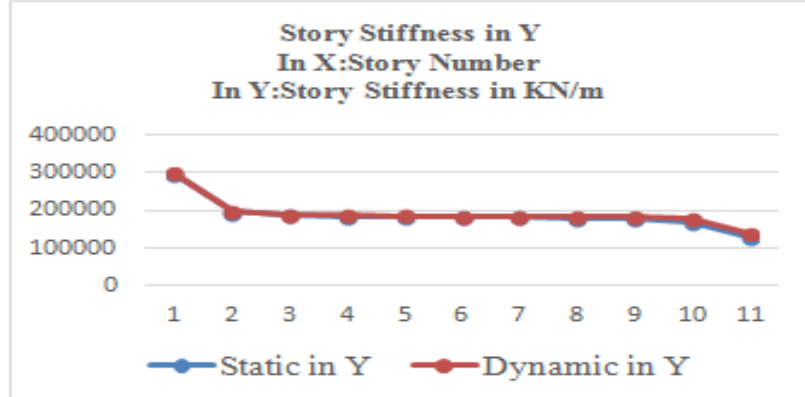

From figure 2.1 and 2.2, it was observed that there was increment in Shear Stiffness by $6.93 \%$ and $7.2 \%$ when compared Static Story Stiffness in X direction with respect to Static Stiffness in Y direction.

\section{BASE SHEAR TABLE}

Table: 2 Linear Static Analysis Base Shear and

Response Spectrum Analysis Base Shear

\begin{tabular}{|c|c|c|c|c|c|}
\hline \multicolumn{3}{|c|}{$\begin{array}{c}\text { Linear Static Analysis } \\
\text { Base Shear }\end{array}$} & \multicolumn{3}{|c|}{$\begin{array}{c}\text { Response Spectrum Analysis } \\
\text { Base Shear }\end{array}$} \\
\hline Case & $\begin{array}{l}\text { Static } \\
\mathbf{X}(\mathbf{K}))\end{array}$ & $\begin{array}{c}\text { Static } \\
\mathbf{Y}(\mathbf{K N})\end{array}$ & Case & $\begin{array}{c}\text { Dynamic } \\
\mathbf{X}(\mathbf{K N})\end{array}$ & $\begin{array}{c}\text { Dynamic } \\
\text { Y(KN) }\end{array}$ \\
\hline Casel & 282.8 & 283.0 & Casel & 283.1 & 283.0 \\
\hline Case2 & 452.4 & 452.8 & Case2 & 452.9 & 452.8 \\
\hline Case 3 & 678.6 & 679.2 & Case3 & 679.4 & 679.2 \\
\hline Case4 & 1017.9 & 1018.8 & Case 4 & 1019.1 & 1018.8 \\
\hline \multicolumn{3}{|c|}{$\begin{array}{l}\text { Base Shear in the } \\
\text { X-Direction }\end{array}$} & \multicolumn{3}{|c|}{$\begin{array}{l}\text { Base Shear in the } \\
\text { Y-Direction }\end{array}$} \\
\hline Case & $\begin{array}{l}\text { Static } \\
\mathbf{X}(\mathrm{KN})\end{array}$ & $\begin{array}{c}\text { Dynamic } \\
\text { X(KN) }\end{array}$ & Case & $\begin{array}{c}\text { Static } \\
\mathbf{Y}(\mathbf{K N})\end{array}$ & $\begin{array}{c}\text { Dynamic } \\
\text { Y(KN) }\end{array}$ \\
\hline Casel & 282.8 & 283.1 & Casel & 283.0 & 283.0 \\
\hline Case2 & 452.4 & 452.9 & Case2 & 452.8 & 452.8 \\
\hline Case 3 & 678.6 & 679.4 & Case3 & 679.2 & 679.2 \\
\hline Case 4 & 1017.9 & 1019.1 & Case 4 & 1018.8 & 1018.8 \\
\hline
\end{tabular}

Figure: 3.1 Linear Static AnalysisBase Shear

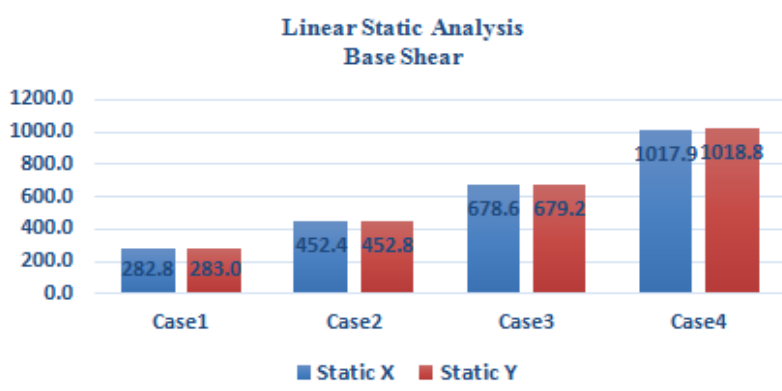

From figure 3.1, it was observed that there was increment in base shear by $0.07 \%, 0.08 \% 0.08 \%$ and $0.08 \%$ when compared Static base shear in $\mathrm{X}$ direction with respect to Static base Shear in Y direction in Case 1, Case 2, Case3 and Case4 respectively.

Figure: 3.2 Response Spectrum Analysis of Base Shear

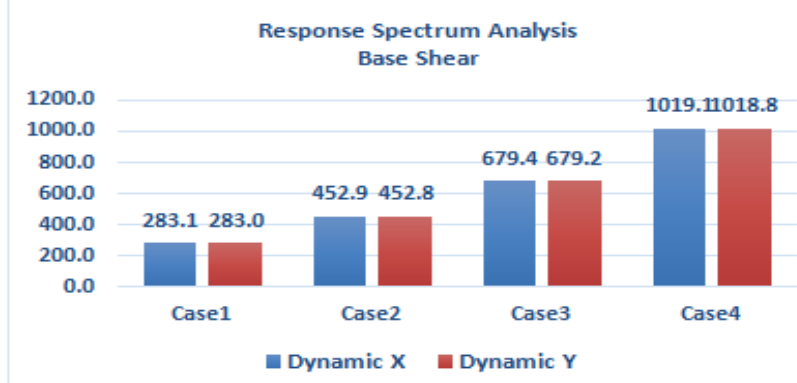

From figure 3.2, it was observed that there was increment in base shear by $0.03 \%, 0.02 \%, 0.03 \%, 0.03 \%$ when compared Dynamic base shear in $\mathrm{X}$ direction with respect to Dynamic base Shear in Y direction in Case1, Case 2 Case 3 and Case4 respectively.

Figure: 3.3 Base Shear in X-direction

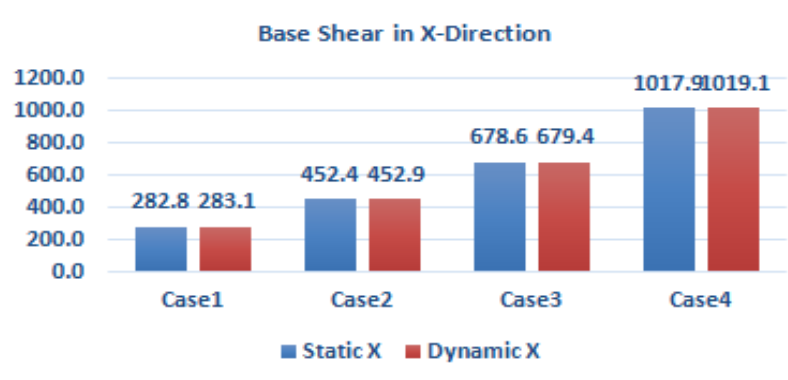

From figure 3.3, it was observed that there was increment in base shear by $0.11 \%$ when compared Dynamic base shear in $\mathrm{X}$ direction with respect to Dynamic base Shear in $\mathrm{Y}$ direction in Case1, Case2, Case 3 and Case4 respectively.

Figure: 3.4 Base Shears in Y-Direction

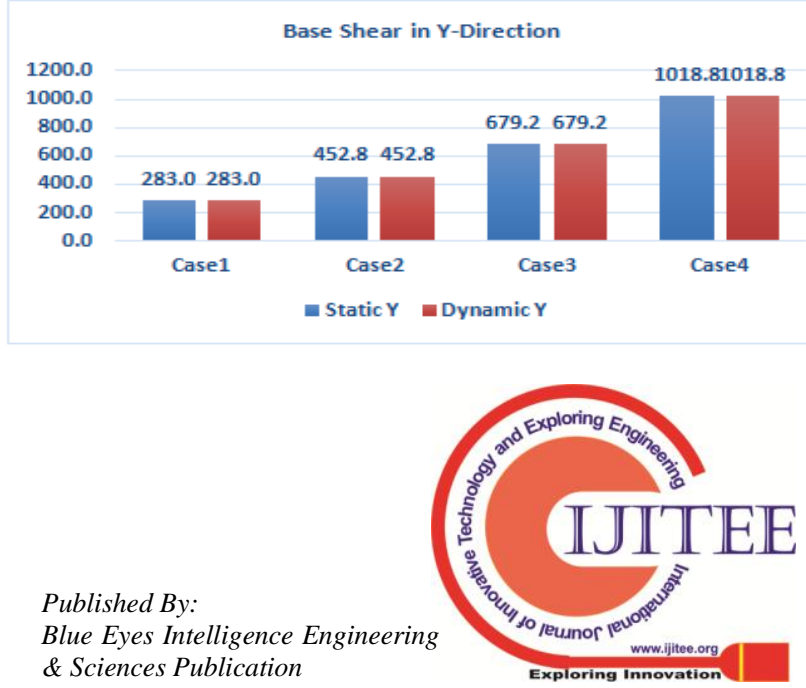


From figure 3.4, it was observed that there was no variation between Static Base shear and Dynamic Base Shear in $\mathrm{Y}$ direction.

\section{STORY SHEAR}

Table: 3.1 Case 1: Story Shear in $X$ and $Y$ Direction

\begin{tabular}{|c|c|c|c|c|}
\hline $\begin{array}{c}\text { Story } \\
\text { shear }\end{array}$ & \multicolumn{2}{|c|}{ X-Direction } & \multicolumn{2}{c|}{ Y-Direction } \\
\hline Story & Static(KN) & Dynamic(KN) & Static(KN) & Dynamic(KN) \\
\hline 1 & $\mathbf{3 0 1 . 7 2 5 8}$ & 298.7295 & 301.9426 & 298.5918 \\
\hline 2 & 299.7061 & 287.1535 & 299.9245 & 287.001 \\
\hline 3 & 295.4686 & 267.788 & 295.6883 & 267.6174 \\
\hline 4 & 287.9683 & 248.0715 & 288.1867 & 247.888 \\
\hline 5 & 276.0293 & 228.7348 & 276.2424 & 228.5623 \\
\hline 6 & 258.4391 & 209.7878 & 258.6419 & 209.6571 \\
\hline 7 & 233.9733 & 190.2676 & 234.1594 & 190.1979 \\
\hline 8 & 201.4033 & 166.7837 & 201.5654 & 166.7728 \\
\hline 9 & 159.4996 & 137.071 & 159.6293 & 137.0936 \\
\hline 10 & 107.033 & 96.2862 & 107.1207 & 96.3135 \\
\hline 11 & 43.7201 & 39.6449 & 43.7563 & 39.6699 \\
\hline
\end{tabular}

Figure 4.1Case 1: Story Shear in $X$ and $Y$ Direction
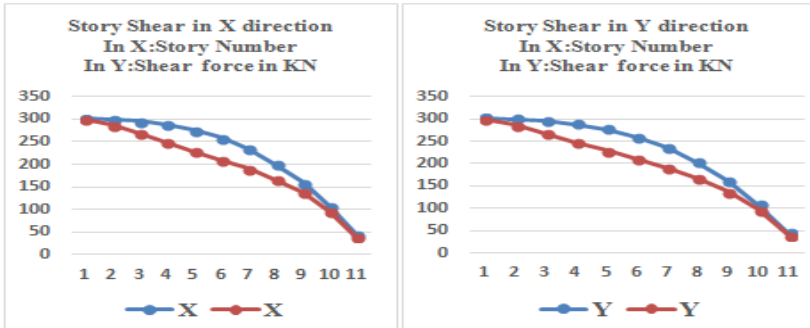
decrement in Story Shear by $23.19 \%, 18.94 \%$ in X-direction and $\mathrm{Y}$-direction respectively in $6^{\text {th }}$ Story when compared to Static Story Shear with respect to Dynamic Story Shear in Case 1.

Table: 3.2 Case 2: Story Shear in $\mathrm{X}$ and Y Direction

\begin{tabular}{|c|c|c|c|c|}
\hline $\begin{array}{l}\text { Story } \\
\text { shear }\end{array}$ & \multicolumn{2}{|c|}{ X-Direction } & \multicolumn{2}{c|}{ Y-Direction } \\
\hline Story & Static(KN) & Dynamic(KN) & Static(KN) & Dynamic(KN) \\
\hline 1 & 482.7612 & 477.9671 & 483.1081 & 477.7469 \\
\hline 2 & 479.5298 & 459.4456 & 479.8793 & 459.2016 \\
\hline 3 & 472.7498 & 428.4608 & 473.1013 & 428.1879 \\
\hline 4 & 460.7493 & 396.9144 & 461.0987 & 396.6208 \\
\hline 5 & 441.6469 & 365.9757 & 441.9879 & 365.6997 \\
\hline 6 & 413.5026 & 335.6604 & 413.827 & 335.4513 \\
\hline 7 & 374.3573 & 304.4281 & 374.655 & 304.3166 \\
\hline 8 & 322.2454 & 266.8539 & 322.5046 & 266.8365 \\
\hline 9 & 255.1994 & 219.3136 & 255.4068 & 219.3498 \\
\hline 10 & 171.2528 & 154.0579 & 171.3931 & 154.1016 \\
\hline 11 & 69.9522 & 63.4319 & 70.01 & 63.4719 \\
\hline
\end{tabular}

Figure 4.2Case 2: Story Shear in $X$ and $Y$ Direction
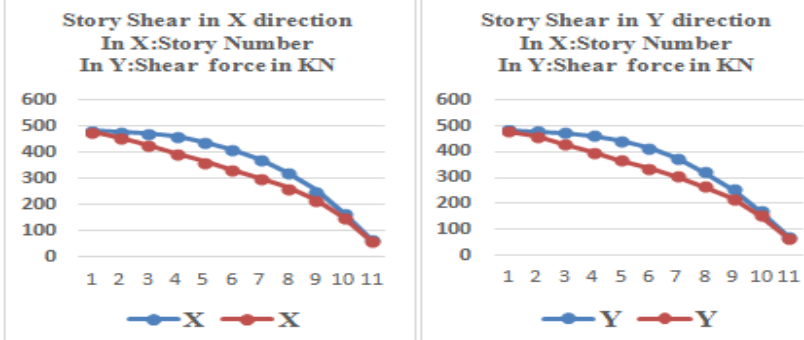

From figure 4.2 , it was observed that there was a decrement in Story Shear by $23.19 \%, 23.36 \%$ in X-direction and $\mathrm{Y}$-direction respectively in $6^{\text {th }}$ Story when compared to Static Story Shear with respect to Dynamic Story Shear in Case 2.
From figure 4.1 , it was observed that there was a

Table: 3.3 Case 3: Story Shear in $\mathrm{X}$ and $\mathrm{Y}$ Direction

\begin{tabular}{|c|c|c|c|c|}
\hline $\begin{array}{c}\text { Story } \\
\text { shear }\end{array}$ & \multicolumn{2}{|c|}{ X-Direction } & \multicolumn{2}{c|}{ Y-Direction } \\
\hline Story & Static(KN) & Dynamic(KN) & Static(KN) & Dynamic(KN) \\
\hline 1 & 724.142 & 716.951 & 724.662 & 716.6203 \\
\hline 2 & 719.295 & 689.168 & 719.819 & 688.8023 \\
\hline 3 & 709.125 & 642.691 & 709.652 & 642.2818 \\
\hline 4 & 691.124 & 595.372 & 691.648 & 594.9312 \\
\hline 5 & 662.47 & 548.964 & 662.982 & $\mathbf{5 4 8 . 5 4 9 6}$ \\
\hline 6 & 620.254 & $\mathbf{5 0 3 . 4 9 1}$ & $\mathbf{6 2 0 . 7 4}$ & $\mathbf{5 0 3 . 1 7 7}$ \\
\hline 7 & $\mathbf{5 6 1 . 5 3 6}$ & $\mathbf{4 5 6 . 6 4 2}$ & $\mathbf{5 6 1 . 9 8 3}$ & $\mathbf{4 5 6 . 4 7 4 9}$ \\
\hline 8 & $\mathbf{4 8 3 . 3 6 8}$ & $\mathbf{4 0 0 . 2 8 1}$ & $\mathbf{4 8 3 . 7 5 7}$ & $\mathbf{4 0 0 . 2 5 4 8}$ \\
\hline 9 & $\mathbf{3 8 2 . 7 9 9}$ & $\mathbf{3 2 8 . 9 7}$ & $\mathbf{3 8 3 . 1 1}$ & $\mathbf{3 2 9 . 0 2 4 7}$ \\
\hline 10 & $\mathbf{2 5 6 . 8 7 9}$ & $\mathbf{2 3 1 . 0 8 7}$ & $\mathbf{2 5 7 . 0 9}$ & $\mathbf{2 3 1 . 1 5 2 3}$ \\
\hline 11 & 104.928 & 95.1478 & 105.015 & 95.2078 \\
\hline
\end{tabular}

Figure 4.3 Case 3: Story Shear in X and Y Direction
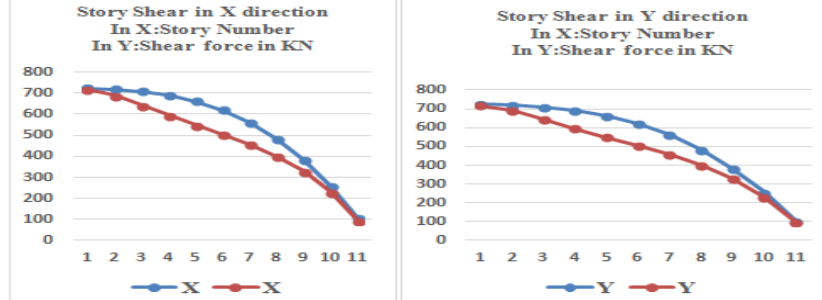

From figure 4.3 , it was observed that there was a decrement in Story Shear by $23.19 \%, 23.36 \%$ in X-direction in Y-direction respectively in $6^{\text {th }}$ Story when compared to Static Story Shear with respect to Dynamic Story Shear in Case 3.

Table: 3.4 Case 4: Story Shear in X and Y Direction

\begin{tabular}{|c|c|c|c|c|}
\hline $\begin{array}{l}\text { Story } \\
\text { shear }\end{array}$ & \multicolumn{2}{|c|}{ X-Direction } & \multicolumn{2}{c|}{ Y-Direction } \\
\hline Story & Static(KN) & Dynamic(KN) & Static(KN) & Dynamic(KN) \\
\hline 1 & 1086.213 & 1075.426 & 1086.993 & 1074.9305 \\
\hline 2 & 1078.942 & 1033.753 & 1079.728 & 1033.2035 \\
\hline 3 & 1063.687 & 964.0368 & 1064.478 & 963.4228 \\
\hline 4 & 1036.686 & $\mathbf{8 9 3 . 0 5 7 4}$ & 1037.472 & $\mathbf{8 9 2 . 3 9 6 8}$ \\
\hline 5 & 993.7055 & $\mathbf{8 2 3 . 4 4 5 3}$ & 994.4727 & $\mathbf{8 2 2 . 8 2 4 4}$ \\
\hline 6 & 930.3809 & 755.2359 & 931.1107 & 754.7655 \\
\hline 7 & $\mathbf{8 4 2 . 3 0 4}$ & 684.9633 & $\mathbf{8 4 2 . 9 7 3 8}$ & $\mathbf{6 8 4 . 7 1 2 3}$ \\
\hline 8 & 725.0521 & 600.4212 & 725.6354 & 600.3822 \\
\hline 9 & 574.1987 & 493.4556 & 574.6653 & 493.5371 \\
\hline 10 & 385.3188 & 346.6302 & 385.6346 & 346.7285 \\
\hline 11 & 157.3924 & 142.7218 & 157.5226 & 142.8117 \\
\hline
\end{tabular}

Figure 4.4 Case 4: Story Shear in $X$ and in $Y$ Direction
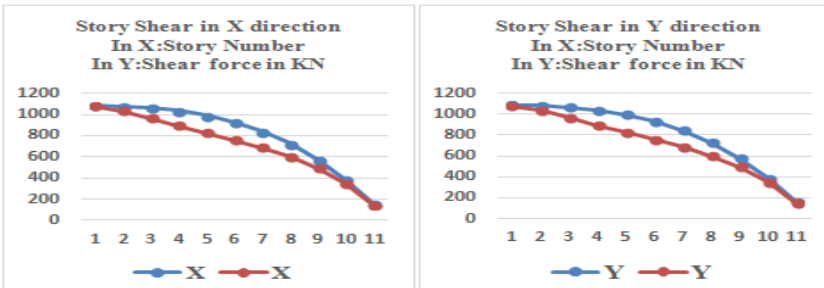

From figure 4.4 , it was observed that there was a decrement in Story Shear by $23.19 \%, 23.36 \%$ in X-direction and Y-direction respectively in $6^{\text {th }}$ Story when compared to Static Story Shear with respect to Dynamic Story Shear in Case 4.

\section{OVERTURNING MOMENT}

Table 4.1 Case 1: Overturning Moment in $X$ and $Y$

\section{Direction}

\begin{tabular}{|c|c|c|c|c|}
\hline & \multicolumn{2}{|c|}{ X-Direction } & \multicolumn{2}{c|}{ Y-Direction } \\
\hline Story & Static(KNm) & Dynamic(KNm) & Static(KNm) & Dynamic(KNmb \\
\hline base & $\mathbf{7 4 0 0 . 5 7 2}$ & $\mathbf{5 8 9 9 . 3 4 6}$ & $\mathbf{7 3 9 4 . 9}$ & $\mathbf{5 9 0 3 . 7 9 3}$ \\
\hline 1 & $\mathbf{6 4 9 4 . 7 4 5}$ & $\mathbf{5 1 1 1 . 3 3 5}$ & $\mathbf{6 4 8 9 . 7 2 3}$ & $\mathbf{5 1 1 5 . 0 0 4}$ \\
\hline 2 & $\mathbf{5 5 9 4 . 9 7 1}$ & $\mathbf{4 3 6 7 . 9 0 4}$ & $\mathbf{5 5 9 0 . 6 0 4}$ & $\mathbf{4 3 7 0 . 7 0 9}$ \\
\hline 3 & $\mathbf{4 7 0 7 . 9 0 6}$ & $\mathbf{3 6 7 1 . 9 0 3}$ & $\mathbf{4 7 0 4 . 1 9 8}$ & $\mathbf{3 6 7 3 . 8 1 1}$ \\
\hline 4 & $\mathbf{3 8 4 3 . 3 4 6}$ & $\mathbf{3 0 1 8 . 8 0 1}$ & $\mathbf{3 8 4 0 . 2 9 4}$ & $\mathbf{3 0 1 9 . 8 7 4}$ \\
\hline 5 & $\mathbf{3 0 1 4 . 6 1 9}$ & $\mathbf{2 4 0 4 . 7 0 5}$ & $\mathbf{3 0 1 2 . 2 0 6}$ & $\mathbf{2 4 0 5 . 1 0 6}$ \\
\hline 6 & $\mathbf{2 2 3 8 . 6 9 3}$ & 1827.864 & $\mathbf{2 2 3 6 . 8 8 8}$ & 1827.83 \\
\hline 7 & 1536.215 & 1292.9 & $\mathbf{1 5 3 4 . 9 6 8}$ & $\mathbf{1 2 9 2 . 6 7 5}$ \\
\hline 8 & 931.5188 & $\mathbf{8 1 2 . 0 8}$ & $\mathbf{9 3 0 . 7 5 8 2}$ & $\mathbf{8 1 1 . 8 4 7 8}$ \\
\hline 9 & $\mathbf{4 5 2 . 6 3 1}$ & $\mathbf{4 0 7 . 1 1 5 5}$ & $\mathbf{4 5 2 . 2 5 9 3}$ & $\mathbf{4 0 6 . 9 5 8 8}$ \\
\hline 10 & 131.2688 & 119.0097 & 131.1603 & 118.9348 \\
\hline 11 & 0 & 0 & 0 & 0 \\
\hline
\end{tabular}


Figure 5.1 Case 1: Overturning Moment in $\mathrm{X}$ and $\mathrm{Y}$ Direction
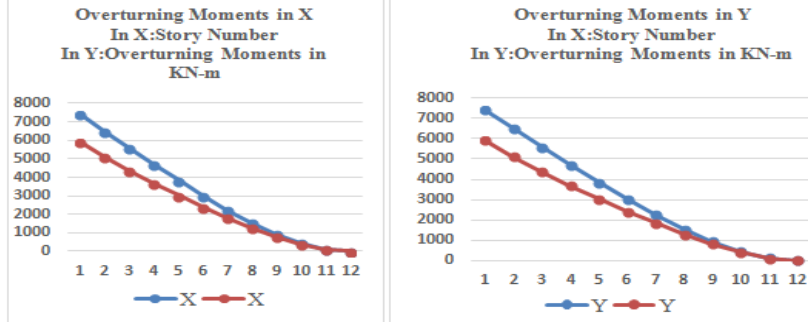

From figure 5.1, it was observed that there was a decrement in Story Overturning Moments by $28.21 \%$, $28.05 \%$ in $\mathrm{X}$-direction and Y-direction respectively in $3^{\text {th }}$ Story when compared to Static Story Overturning Moments with respect to Dynamic Story Overturning Moments in Case1.

Table 4.2 Case 2: Overturning Moment in $\mathrm{X}$ and $\mathrm{Y}$ Direction

\begin{tabular}{|c|c|c|c|c|}
\hline & \multicolumn{2}{|c|}{ X-Direction } & \multicolumn{2}{c|}{ Y-Direction } \\
\hline Story & Static(KNm) & Dynamic(KNm) & Static(KNm) & Dynamic(KNm) \\
\hline base & 11840.9 & 9438.95 & 11831.8 & 9446.07 \\
\hline 1 & 10391.6 & $\mathbf{8 1 7 8 . 1 4}$ & 10383.6 & $\mathbf{8 1 8 4 . 0 1}$ \\
\hline 2 & $\mathbf{8 5 1 . 9 5}$ & 6988.65 & $\mathbf{8 9 4 4 . 9 7}$ & 6993.13 \\
\hline 3 & 7532.65 & 5875.04 & 7526.72 & 5878.1 \\
\hline 4 & 6149.35 & 4830.08 & 6144.47 & 4831.8 \\
\hline 5 & 4823.39 & $\mathbf{3 8 4 7 . 5 3}$ & 4819.53 & $\mathbf{3 8 4 8 . 1 7}$ \\
\hline 6 & $\mathbf{3 5 8 1 . 9 1}$ & 2924.58 & $\mathbf{3 5 7 9 . 0 2}$ & $\mathbf{2 9 2 4 . 5 3}$ \\
\hline 7 & 2457.94 & 2068.64 & $\mathbf{2 4 5 5 . 9 5}$ & 2068.28 \\
\hline 8 & 1490.43 & 1299.33 & 1489.21 & 1298.96 \\
\hline 9 & 724.21 & 651.385 & 723.615 & 651.134 \\
\hline 10 & 210.03 & 190.416 & 209.857 & 190.296 \\
\hline 11 & 0 & 0 & 0 & 0 \\
\hline
\end{tabular}

Figure 5.2 Case 2: Overturning Moment in $\mathrm{X}$ and $\mathrm{Y}$ Direction
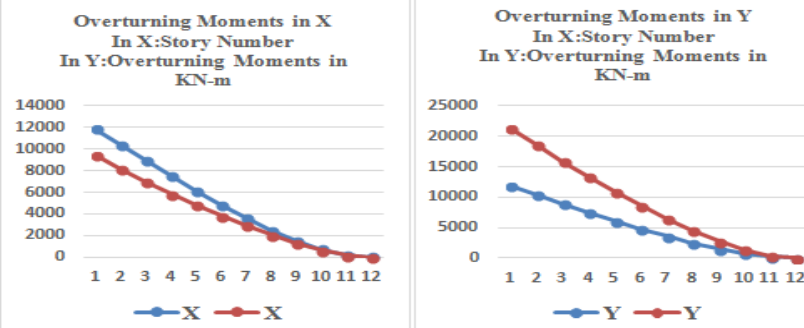

From figure 5.2, it was observed that there was a decrement in Story Overturning Moments by $28.21 \%$ in $\mathrm{X}$ direction and increment by $28.05 \%$ Y-direction respectively in $3^{\text {th }}$ Story when compared to Static Story Overturning Moments with respect to Dynamic Story Overturning Moments in Case2.

Table 4.3 Case 3: Overturning Moment in $\mathrm{X}$ and $\mathrm{Y}$ Direction

\begin{tabular}{|c|c|c|c|c}
\hline & \multicolumn{2}{|c|}{ X-Direction } & \multicolumn{2}{c}{ Y-Direction } \\
\hline Story & Static(KNm) & Dynamic(KNm) & Static(KNm) & Dynamic(KNm) \\
\hline base & 17761.4 & 14158.4 & 17747.8 & 14169.1 \\
\hline 1 & 15587.4 & 12267.2 & 15575.3 & 12276 \\
\hline 2 & 13427.9 & 10483 & 13417.5 & 10489.7 \\
\hline 3 & 11299 & $\mathbf{8 8 1 2 . 5 7}$ & 11290.1 & $\mathbf{8 8 1 7 . 1 5}$ \\
\hline 4 & 9224.03 & 7245.12 & 9216.7 & 7247.7 \\
\hline 5 & 7235.08 & 5771.29 & 7229.29 & 5772.26 \\
\hline 6 & 5372.86 & 4386.87 & 5368.53 & 4386.79 \\
\hline 7 & $\mathbf{3 6 8 6 . 9 2}$ & $\mathbf{3 1 0 2 . 9 6}$ & $\mathbf{3 6 8 3 . 9 2}$ & $\mathbf{3 1 0 2 . 4 2}$ \\
\hline 8 & 2235.65 & 1948.99 & 2233.82 & 1948.43 \\
\hline 9 & 1086.31 & 977.077 & 1085.42 & 976.701 \\
\hline 10 & 315.045 & 285.623 & 314.785 & 285.444 \\
\hline 11 & 0 & 0 & 0 & 0 \\
\hline
\end{tabular}

Figure 5.3 Case 3: Overturning Moment in $\mathrm{X}$ and $\mathrm{Y}$ Direction
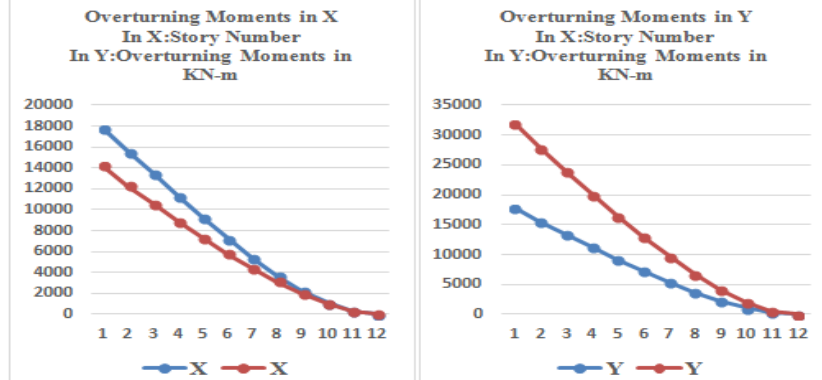

From figure 5.3, it was observed that there was a decrement in Story Overturning Moments by $28.2 \%$ in Xdirection and increment by $28.05 \%$ Y-direction respectively in $3^{\text {th }}$ Story when compared to Static Story Overturning Moments with respect to Dynamic Story Overturning Moments in Case 3.

Table 4.4 Case 4: Overturning Moment in $X$ and $Y$ Direction

\begin{tabular}{|c|c|c|c|c|}
\hline & \multicolumn{2}{|c|}{ X-Direction } & \multicolumn{2}{c|}{ Y-Direction } \\
\hline Story & Static(KNm) & Dynamic(KNm) & Static(KNm) & Dynamic(KNm) \\
\hline base & 26642.1 & 21237.6 & 21237.6 & 21253.7 \\
\hline 1 & 23381.1 & 18400.8 & 18400.8 & 18414 \\
\hline 2 & 20141.9 & 15724.5 & 15724.5 & 15734.6 \\
\hline 3 & 16948.5 & 13218.8 & 13218.8 & 13225.7 \\
\hline 4 & 13836 & 10867.7 & 10867.7 & 10871.5 \\
\hline 5 & 10852.6 & $\mathbf{8 6 5 6 . 9 4}$ & $\mathbf{8 6 5 6 . 9 4}$ & $\mathbf{8 6 5 8 . 3 8}$ \\
\hline 6 & $\mathbf{8 0 5 9 . 2 9}$ & 6580.31 & 6580.31 & 6580.19 \\
\hline 7 & 5530.37 & 4654.44 & 4654.44 & 4653.63 \\
\hline 8 & 3353.47 & 2923.49 & 2923.49 & 2922.65 \\
\hline 9 & 1629.47 & 1465.62 & 1465.62 & 1465.05 \\
\hline 10 & 472.568 & 428.435 & 428.435 & 428.165 \\
\hline 11 & 0 & 0 & 0 & 0 \\
\hline
\end{tabular}

Figure 5.4 Case 4: Overturning Moment in $X$ and $Y$ Direction
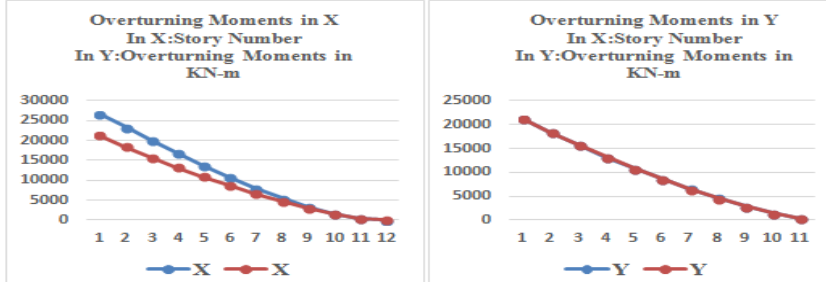

From figure 5.4, it was observed that there was a decrement in Story Overturning Moments by $28.21 \%$ in Xdirection and increment by $0.075 \%$ Y-direction respectively in $3^{\text {th }}$ Story when compared to Static Story Overturning Moments with respect to Dynamic Story Overturning Moments in Case3.

\section{MAXIMUM STORY DISPLACEMENT}

Table 5.1 Case 1: Maximum Story Displacement in $X$ and Y Direction

\begin{tabular}{|c|c|c|c|c|}
\hline & \multicolumn{2}{|c|}{ X-Direction } & \multicolumn{2}{c|}{ Y-Direction } \\
\hline Story & Static(mm) & Dynamic(mm) & Static(mm) & Dynamic(mm) \\
\hline 1 & 0 & 0 & 0 & 0 \\
\hline 2 & 2.486 & 2.558 & 2.497 & 2.553 \\
\hline 3 & 3.911 & 4.137 & 3.93 & 4.129 \\
\hline 4 & 5.221 & 5.693 & 5.249 & 5.684 \\
\hline 5 & 6.403 & 7.195 & 6.44 & 7.185 \\
\hline 6 & 7.453 & 8.61 & 7.498 & 8.6 \\
\hline 7 & 8.366 & 9.9 & 8.419 & 9.89 \\
\hline 8 & 9.131 & 11.019 & 9.192 & 11.011 \\
\hline 9 & 9.729 & 11.92 & 9.798 & 11.914 \\
\hline 10 & 10.139 & 12.549 & 10.214 & 12.546 \\
\hline 11 & 10.356 & 12.888 & 10.438 & 12.889 \\
\hline
\end{tabular}


Figure 6.1 Case 1: Maximum Story Displacement in X and Y Direction

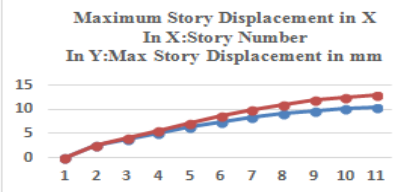

$\rightarrow x \rightarrow x$

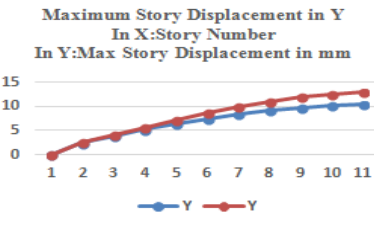

From figure 6.1 , it was observed that there was an increment in Maximum Story Displacement by $24.45 \%$, $23.48 \%$ in $\mathrm{X}$-direction and Y-direction respectively in $11^{\text {th }}$ Story when compared to Static Maximum Story Displacement with respect to Dynamic Maximum Story Displacement in Case1.

Table 5.2 Case 2: Maximum Story Displacement in $\mathrm{X}$ and Y Direction

\begin{tabular}{|c|c|c|c|c|}
\hline & \multicolumn{2}{|c|}{ X-Direction } & \multicolumn{2}{c|}{ Y-Direction } \\
\hline Story & Static(mm) & Dynamic(mm) & Static(mm) & Dynamic(mm) \\
\hline 1 & 0 & 0 & 0 & 0 \\
\hline 2 & 4.093 & 3.977 & 4.085 & 3.996 \\
\hline 3 & 6.619 & 6.257 & 6.607 & 6.289 \\
\hline 4 & 9.109 & 8.353 & 9.094 & 8.398 \\
\hline 5 & 11.513 & 10.245 & 11.496 & 10.303 \\
\hline 6 & 13.776 & 11.925 & 13.76 & 11.997 \\
\hline 7 & 15.839 & 13.386 & 15.824 & 13.471 \\
\hline 8 & 17.631 & 14.609 & 17.618 & 14.708 \\
\hline 9 & 19.071 & 15.567 & 19.062 & 15.677 \\
\hline 10 & 20.078 & 16.222 & 20.074 & 16.343 \\
\hline 11 & 20.62 & 16.57 & 20.622 & 16.7 \\
\hline
\end{tabular}

Figure 6.2 Case 2: Maximum Story Displacement in $\mathrm{X}$ and Y Direction
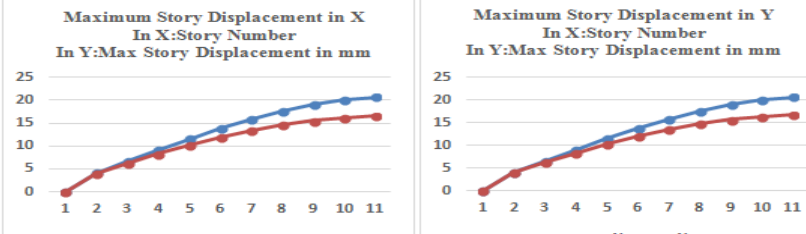

$\rightarrow x \rightarrow x$

$\rightarrow r \rightarrow$

From figure 6.2, it was observed that there was a decrement in Maximum Story Displacement by $24.44 \%$, $23.49 \%$ in X-direction and Y-direction respectively in $11^{\text {th }}$ Story when compared to Static Maximum Story Displacement with respect to Dynamic Maximum Story Displacement in Case2.

Table 5.3 Case 3: Maximum Story Displacement in X and Y Direction

\begin{tabular}{|c|c|c|c|c|}
\hline & \multicolumn{2}{|c|}{ X-Direction } & \multicolumn{2}{c|}{ Y-Direction } \\
\hline Story & Static(mm) & Dynamic(mm) & Static(mm) & Dynamic(mm) \\
\hline 1 & 0 & 0 & 0 & 0 \\
\hline 2 & 6.14 & 5.966 & 6.128 & 5.994 \\
\hline 3 & 9.929 & 9.386 & 9.911 & 9.433 \\
\hline 4 & 13.664 & 12.53 & 13.642 & 12.597 \\
\hline 5 & 17.269 & 15.367 & 17.244 & 15.455 \\
\hline 6 & 20.664 & 17.888 & 20.64 & 17.996 \\
\hline 7 & 23.759 & 20.078 & 23.736 & 20.207 \\
\hline 8 & 26.447 & 21.914 & 26.427 & 22.062 \\
\hline 9 & 28.607 & 23.35 & 28.593 & 23.516 \\
\hline 10 & 30.117 & 24.333 & 30.111 & 24.515 \\
\hline 11 & 30.931 & 24.855 & 30.933 & 25.051 \\
\hline
\end{tabular}

Figure 6.3 Case 3: Maximum Story Displacement in $\mathrm{X}$ and Y Direction

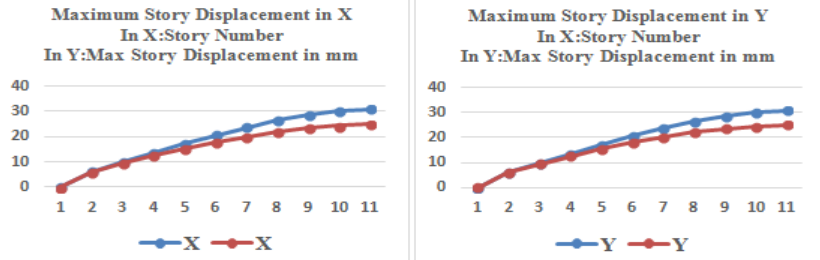

From figure 6.3, it was observed that there was a decrement in Maximum Story Displacement by $24.45 \%$,
$23.48 \%$ in $\mathrm{X}$-direction and $\mathrm{Y}$-direction respectively in $11^{\text {th }}$ Story when compared to Static Maximum Story Displacement with respect to Dynamic Maximum Story Displacement in Case3.

Table 5.4 Case 4: Maximum Story Displacement in X and Y Direction

\begin{tabular}{|c|c|c|c|c|}
\hline & \multicolumn{2}{|c|}{ X-Direction } & \multicolumn{2}{c|}{ Y-Direction } \\
\hline Story & Static(mm) & Dynamic(mm) & Static(mm) & Dynamic(mm) \\
\hline 1 & 0 & 0 & 0 & 0 \\
\hline 2 & 9.209 & $\mathbf{8 . 9 4 9}$ & 9.191 & $\mathbf{8 . 9 9 1}$ \\
\hline 3 & 14.893 & 14.079 & 14.866 & 14.15 \\
\hline 4 & 20.496 & 18.795 & 20.462 & 18.896 \\
\hline 5 & 25.903 & 23.051 & 25.866 & 23.183 \\
\hline 6 & 30.997 & 26.831 & 30.959 & 26.994 \\
\hline 7 & 35.639 & 30.117 & 35.604 & 30.31 \\
\hline 8 & 39.67 & 32.871 & 39.641 & 33.093 \\
\hline 9 & 42.911 & 35.025 & 42.89 & 35.274 \\
\hline 10 & 45.176 & 36.499 & 45.167 & 36.772 \\
\hline 11 & 46.396 & 37.282 & 46.4 & 37.576 \\
\hline
\end{tabular}

Figure 6.4 Case 4: Maximum Story Displacement in X and Y Direction
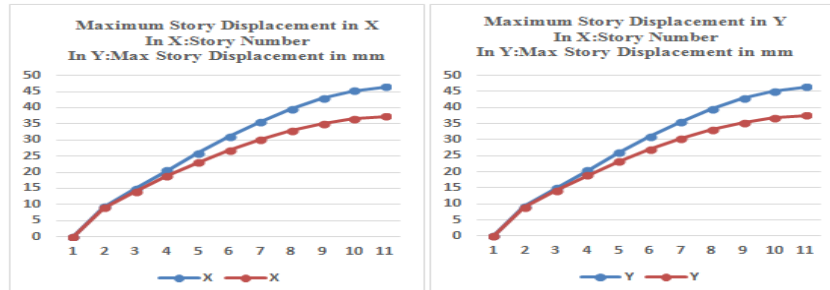

From figure 6.4 , it was observed that there was a decrement in Maximum Story Displacement by $24.45 \%$, $23.48 \%$ in $\mathrm{X}$-direction and Y-direction respectively in $11^{\text {th }}$ Story when compared to Static Maximum Story Displacement with respect to Dynamic Maximum Story Displacement in Case4.

\section{STORY DRIFT}

Table 6.1 Case 1: Story Drift in $X$ and $Y$ Direction

\begin{tabular}{|c|c|c|c|c|}
\hline & \multicolumn{2}{|c|}{ X-Direction } & \multicolumn{2}{c|}{ Y-Direction } \\
\hline Story & Static(mm) & Dynamic(mm) & Static(mm) & Dynamic(mm) \\
\hline 1 & 1.024 & 1.004 & 1.023 & 1.001 \\
\hline 2 & 1.528 & 1.449 & 1.525 & 1.444 \\
\hline 3 & 1.574 & 1.417 & 1.571 & 1.412 \\
\hline 4 & 1.551 & 1.329 & 1.549 & 1.325 \\
\hline 5 & 1.496 & 1.234 & 1.495 & 1.231 \\
\hline 6 & 1.409 & 1.139 & 1.409 & 1.137 \\
\hline 7 & 1.284 & 1.036 & 1.285 & 1.035 \\
\hline 8 & 1.115 & 0.912 & 1.117 & 0.912 \\
\hline 9 & 0.897 & 0.752 & 0.899 & 0.753 \\
\hline 10 & 0.626 & 0.538 & 0.63 & 0.539 \\
\hline 11 & 0.336 & 0.283 & 0.339 & 0.286 \\
\hline
\end{tabular}

Figure 7.1 Case 1: Story Drift in $X$ and $Y$ Direction
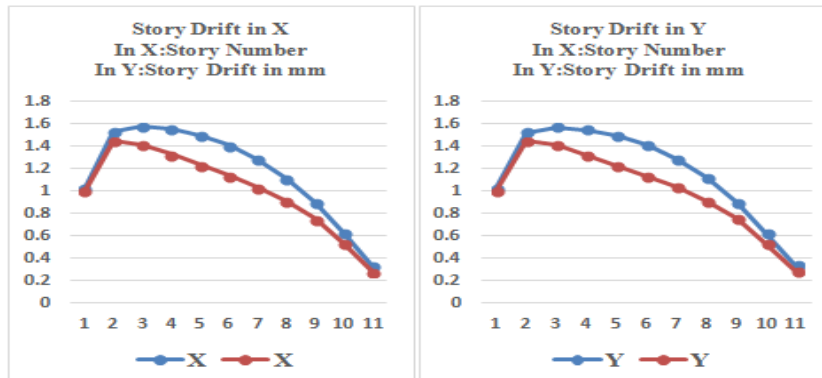

From figure 7.1, it was observed that there was a decrement in Story Drift by $19.65 \%, 19.02 \%$ in X-direction and Y-direction respectively in $7^{\text {th }}$ Story when compared to Static Story Drift with respect to Dynamic Story Drift in Case1. 
Table 6.2 Case 2: Story Drift in X and Y Direction

\begin{tabular}{|c|c|c|c|c|}
\hline & \multicolumn{2}{|c|}{ X-Direction } & \multicolumn{2}{c|}{ Y-Direction } \\
\hline Story & Static(mm) & Dynamic(mm) & Static(mm) & Dynamic(mm) \\
\hline 1 & 1.639 & 1.607 & 1.636 & 1.602 \\
\hline 2 & 2.445 & 2.318 & 2.44 & 2.31 \\
\hline 3 & 2.518 & 2.267 & 2.514 & 2.259 \\
\hline 4 & 2.481 & 2.126 & 2.479 & 2.119 \\
\hline 5 & 2.394 & 1.975 & 2.393 & 1.97 \\
\hline 6 & 2.255 & 1.822 & 2.255 & 1.819 \\
\hline 7 & 2.055 & 1.658 & 2.056 & 1.657 \\
\hline 8 & 1.784 & 1.459 & 1.787 & 1.459 \\
\hline 9 & 1.434 & 1.203 & 1.438 & 1.204 \\
\hline 10 & 1.002 & 0.861 & 1.007 & 0.863 \\
\hline 11 & 0.537 & 0.454 & 0.543 & 0.457 \\
\hline
\end{tabular}

Fig 7.2 Case 2: Story Drift in X and Y Direction

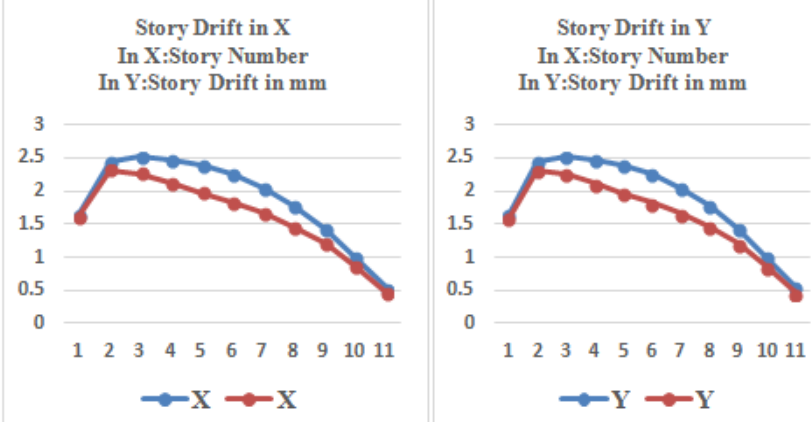

From figure 7.2, it was observed that there was a decrement in Story Drift by $23.95 \%, 24.08 \%$ in X-direction and $\mathrm{Y}$-direction respectively in $7^{\text {th }}$ Story when compared to Static Story Drift with respect to Dynamic Story Drift in Case2.

Table 6.3 Case 3: Story Drift in X and Y Direction

\begin{tabular}{|c|c|c|c|c|}
\hline & \multicolumn{2}{|c|}{ X-Direction } & \multicolumn{2}{c|}{ Y-Direction } \\
\hline Story & Static(mm) & Dynamic(mm) & Static(mm) & Dynamic(mm) \\
\hline 1 & 3.687 & 3.615 & 3.681 & 3.604 \\
\hline 2 & 5.501 & 5.216 & 5.489 & 5.198 \\
\hline 3 & 5.666 & 5.101 & 5.656 & 5.083 \\
\hline 4 & 5.583 & 4.783 & 5.577 & 4.768 \\
\hline 5 & 5.386 & 4.443 & 5.383 & 4.432 \\
\hline 6 & 5.073 & 4.099 & 5.073 & 4.092 \\
\hline 7 & 4.623 & 3.731 & 4.626 & 3.728 \\
\hline 8 & 4.015 & 3.283 & 4.02 & 3.283 \\
\hline 9 & 3.228 & 2.707 & 3.236 & 2.71 \\
\hline 10 & 2.255 & 1.936 & 2.266 & 1.942 \\
\hline 11 & 1.209 & 1.021 & 1.222 & 1.028 \\
\hline
\end{tabular}

Figure 7.3 Case 3: Story Drift in $X$ and $Y$ Direction

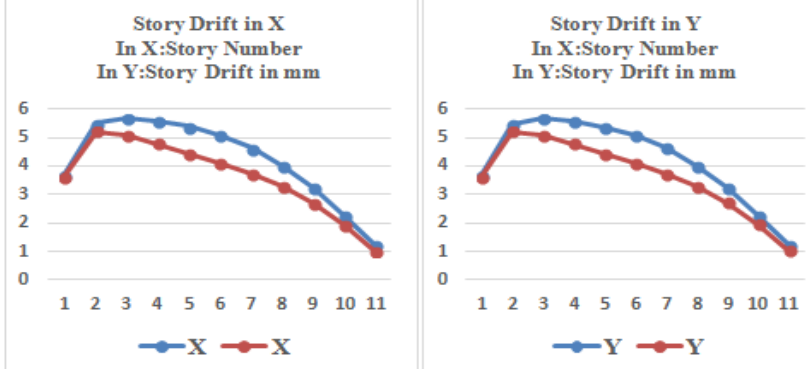

From figure 7.3 , it was observed that there was a decrement in Story Drift by $23.91 \%, 24.09 \%$ in X-direction and $\mathrm{Y}$-direction respectively in $7^{\text {th }}$ Story when compared to Static Story Drift with respect to Dynamic Story Drift in Case3.
Table 6.4 Case 4: Story Drift in $X$ and $Y$ Direction

\begin{tabular}{|c|c|c|c|c|}
\hline & \multicolumn{2}{|c|}{ X-Direction } & \multicolumn{2}{c|}{ Y-Direction } \\
\hline Story & Static(mm) & Dynamic(mm) & Static(mm) & Dynamic(mm) \\
\hline 1 & 3.687 & 3.615 & 3.681 & 3.604 \\
\hline 2 & 5.501 & 5.216 & 5.489 & 5.198 \\
\hline 3 & 5.666 & 5.101 & 5.656 & 5.083 \\
\hline 4 & 5.583 & 4.783 & 5.577 & 4.768 \\
\hline 5 & 5.386 & 4.443 & 5.383 & 4.432 \\
\hline 6 & 5.073 & 4.099 & 5.073 & 4.092 \\
\hline 7 & 4.623 & 3.731 & 4.626 & 3.728 \\
\hline 8 & 4.015 & 3.283 & 4.02 & 3.283 \\
\hline 9 & 3.228 & 2.707 & 3.236 & 2.71 \\
\hline 10 & 2.255 & 1.936 & 2.266 & 1.942 \\
\hline 11 & 1.209 & 1.021 & 1.222 & 1.028 \\
\hline
\end{tabular}

Figure 7.4 Case 4: Story Drift in $X$ and $Y$ Direction
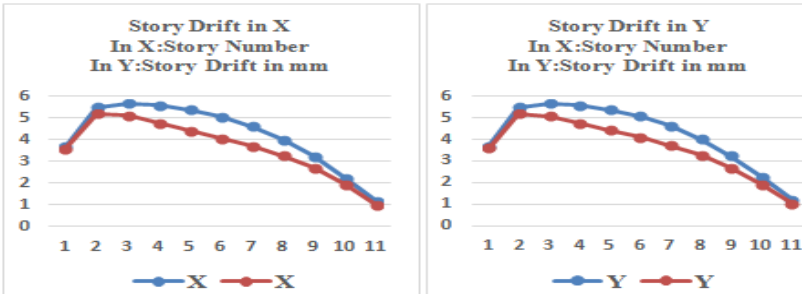

From figure 7.4, it was observed that there was a decrement in Story Drift by $23.91 \%, 24.09 \%$ in X-direction and Ydirection respectively in $7^{\text {th }}$ Story when compared to Static Story Drift with respect to Dynamic Story Drift in Case4.
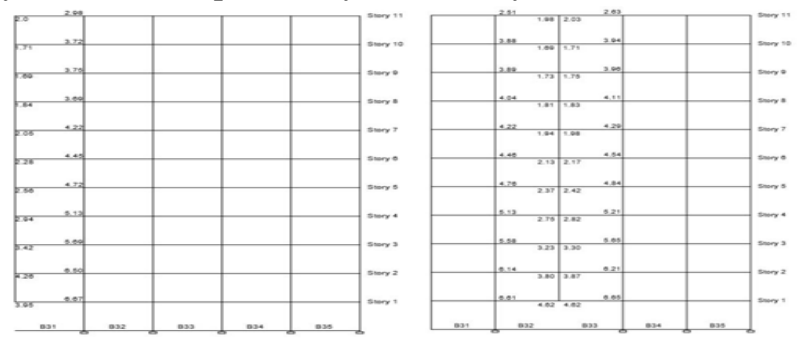

From the figure 8.1 and 8.2 , it was observed that there was continuous decrease in DCR value from bottom Story to top Story which concludes that the failure is more at bottom Story than top Story.
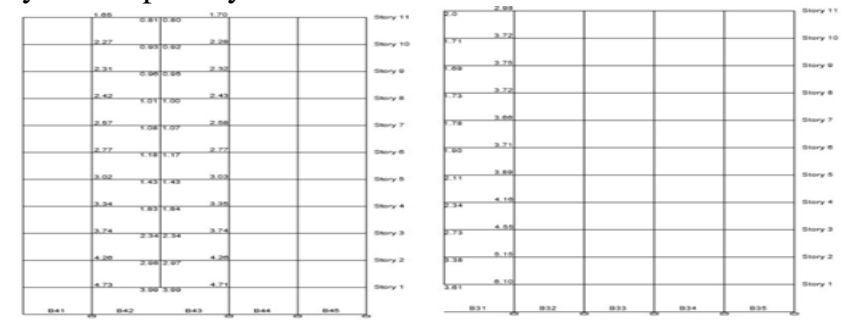

From the figure 8.3 and 8.4 , it was observed that there was continuous decrease in DCR value from bottom Story to top Story which concludes that the failure is more at bottom Story than top Story and it was also found that in figure 8.3, the $11^{\text {th }}$ Story beam was safe against Progressive Collapse. 

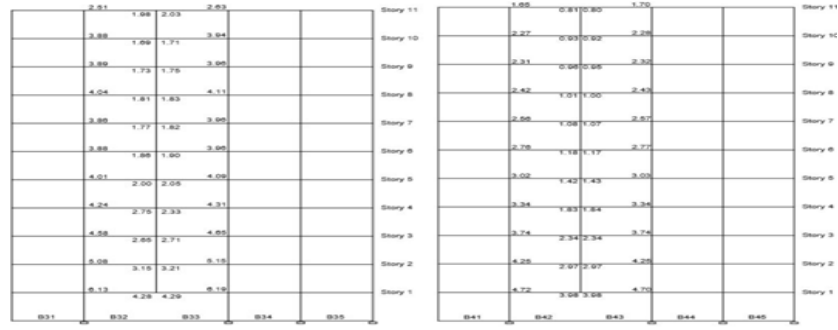

Figure 8.5 Case 2: Exterior Column Removal Figure 8.6 Case 2: Interior Column Removal

From the figure 8.5 and 8.6 , it was observed that there was continuous decrease in DCR value from bottom Story to top Story which concludes that the failure is more at bottom Story than top Story and it was also found that in figure 8.6, the $11^{\text {th }}$ Story beam was safe against Progressive Collapse.
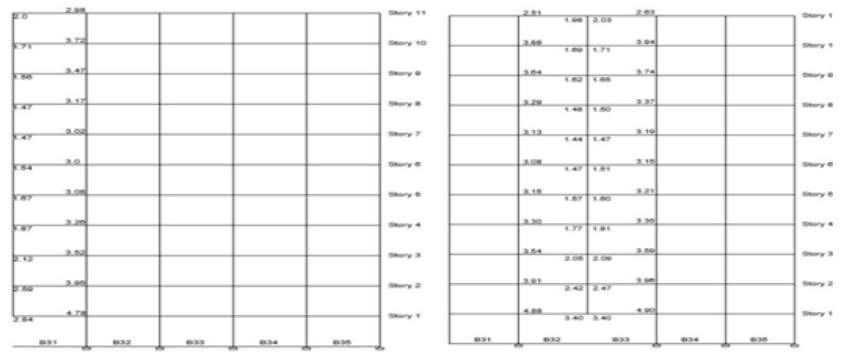

Figure 8.7 Case 3: Corner Column Removal Figure 8.8 Case 3: Exterior Column Removal

From the figure 8.7 and 8.8 , it was observed that there was continuous decrease in DCR value from bottom Story to top Story which concludes that the failure is more at bottom Story than top Story.
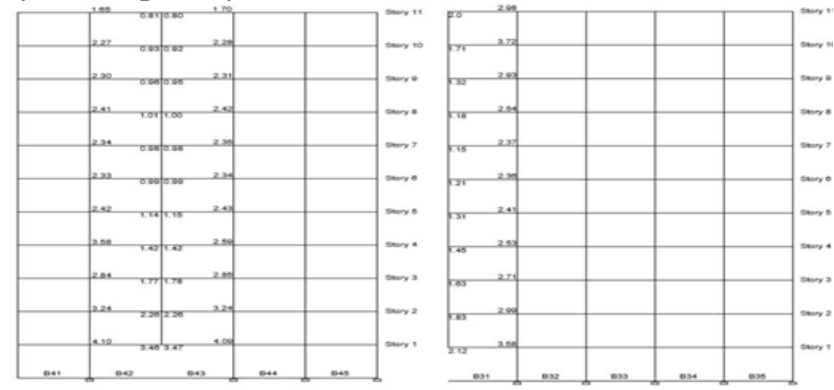

Figure 8.9 Case 3: Interior Column Removal Figure 8.10 Case 4: Corner Column Remov

From the figure 8.9 and 8.10 , it was observed that there was continuous decrease in DCR value from bottom Story to top Story which concludes that the failure is more at bottom Story than top Story and it was also found that in figure 8.9, the $11^{\text {th }}$ Story beam was safe against Progressive Collapse.
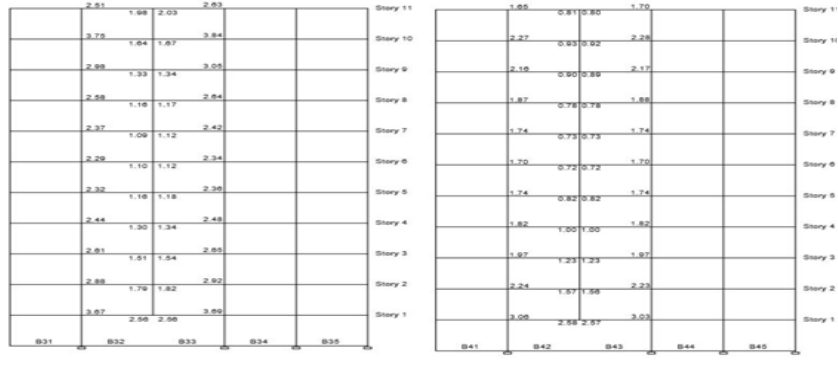

Figure 8.11 Case 4: Exterior Column Removal Figure 8.12 Case 4: Interior Column Removal

From the figure 8.11 and 8.12 , it was observed that there was continuous decrease in DCR value from bottom Story to top Story which concludes that the failure is more at bottom Story than top Story and it was also found that in figure 8.12 , the $3^{\text {rd }}$, 4th, 5th, 6th, 7th, 8th, 11th Story beam was safe against Progressive Collapse.

\section{CONCLUSION}

After doing Static, Dynamic and progressive Analysis following conclusion were made:

\section{For Static and Dynamic Result:}

There is an increment in Story Stiffness by $6.93 \%$, $7.2 \%$ in $\mathrm{X}$ and $\mathrm{Y}$-direction respectively when compared to Static Story Stiffness with respect to its Dynamic Story Stiffness.

- The maximum variation in Linear Base Shear is by $0.08 \%$ and in Dynamic Base Shear is by $0.03 \%$

- There is a decrement in Story Shear by $23.19 \%$, $23.36 \%$ in $\mathrm{X}$-direction and $\mathrm{Y}$-direction respectively when compared to Static Story Shear with respect to its Dynamic Story Shear.

The maximum variation of Story Overturning Moments is by $28.21 \%, 28.05 \%$ in X-direction and Ydirection respectively when compared to Static Story Overturning Moments with respect to its Dynamic Story Overturning Moments.

The maximum variation of Maximum Story Displacement is by $24.45 \%, 24.49 \%$ in X-direction and Y-direction respectively when compared to Static Maximum Story Displacement with respect to its Dynamic Maximum Story Displacement.

- There is a decrement in Story Drift by $23.95 \%$, $24.09 \%$ in $\mathrm{X}$-direction and Y-direction respectively when compared to Static Story Drift with respect to its Dynamic Story Drift.

\section{For Progressive Collapse Result:}

It was observed that there is continuous decrease in DCR value from bottom Story to top Story, which conclude that the failure is more at bottom Story than top Story and it was also found that in Case 1,Case 2 and Case 3 for Interior Column Removal Case, beam in the $11^{\text {Th }}$ Story is safe against PC.

It was also observed that in Case 4 of Interior Column Removal Case, beam in $3^{\text {rd }}, 4^{\text {th }}, 5^{\text {th }}, 6^{\text {th }}, 7^{\text {th }}, 8^{\text {th }}$ and $11^{\text {th }}$ Story were safe against Progressive Collapse.

The Special Moment Resistance Frame (SMRF) by IS 456:2000 does not provide resistance to progressive Collapse. The SMRF frames are designed for lateral loads and PC failure on these frames due to gravity load.

\section{REFERENCES}

1. General Services Administration (GSA). (2003).Progressive collapse analysis and design guidelines for New federal office buildings and major modernization projects, GSA.

2. Reference ETABS 2015. Version 15, Extended 3-D Analysis of Building Systems, Computers and Structures, Inc.,Berkeley.

3. Mrs. Mir Sana Fatema, Prof. A.A. Hamane "Progressive Collapse of Reinforced Concrete" in International Journal of Emerging Trends in Science and Technology .IJETST, Vol.||03||Issue||12||Pages,4846-4854||December||ISSN, 23489480. 
4. GirumMindaye, Dr. ShaikYajdani "Seismic Analysis of a Multistory RC Frame Building in Different Seismic Zones"in International Journal of Innovative Research in Science, Engineering and Technology, Vol. 5, Issue 9, September 2016.

5. B. Srikanth, V. Ramesh "Comparative Study of Seismic Response for Seismic Coefficient and Response Spectrum Methods" in International Journal of Engineering Research and Applications, ISSN: 2248-9622, Vol. 3, Issue 5, Sep-Oct 2013, pp.1919-1924.

\section{AUTHORS PROFILE}

PONNANA RAMPRASAD, M.Tech(Ph.D), Department of Civil engineering, Assistant Professor, Aditya Institute of Technology \& Management, K.Kotturu, India.

MADHUSMITA MOHARANA, M.Tech, Department of Civil engineering, Aditya Institute of Technology \& Management, K.Kotturu, India.

CH. CHANDRA MOULI, M.Tech(Ph.D), Department of Civil engineering, Snr. Assistant Professor, Aditya Institute of Technology \& Management, K.Kotturu, India. 